\title{
Efecto de Diferentes Aditivos sobre el Comportamiento Reológico de Masas de Harina de Plátano Dominico Hartón (Musa paradisiaca L.)
}

\author{
Jairo Montoya ${ }^{(1) \star}$, German A. Giraldo(2) y Leidy T. Sánchez ${ }^{(2)}$ \\ (1) Facultad de Ciencias Básicas y Tecnologías, Programa de Química, Universidad del Quindío, \\ Armenia-Colombia. (e-mail: jmontoya@uniquindio.edu.co) \\ (2) Facultad de Ciencias Agroindustriales, Programa de Ingeniería de Alimentos, Universidad del Quindío, \\ Armenia-Colombia. (e-mail: ggiraldo@uniquindio.edu.co; Itsanchez@uniquindio.edu.co)
}

* Autor a quien debe ser dirigida la correspondencia

Recibido Ago. 23, 2018; Aceptado Nov. 20, 2018; Versión final Ene. 20, 2019, Publicado Ago. 2019

\begin{abstract}
Resumen
El objetivo de este trabajo fue evaluar el efecto de diferentes aditivos en el comportamiento reológico de masas de harina de plátano nativa para la formulación de una harina funcional libre de gluten. Este comportamiento fue analizado a través de parámetros como módulo elástico (G'), módulo viscoso (G"), tangente de pérdida (tan $\delta$ ) y viscosidad aparente. La elección de la formulación más adecuada se realizó por medio de un diseño de experimentos completamente al azar por comparación con el control (harina de trigo). La proteína de huevo, el emulsificador DATEM y la goma xántan generaron parámetros viscoelásticos más parecidos al control. La formulación de la masa de harina funcional; más similar al control fue aquella con concentraciones intermedias de los aditivos elegidos. Se concluye que el efecto de los aditivos sobre el comportamiento reológico de las masas de harina de plátano depende de la naturaleza química del aditivo y de la composición de la harina.
\end{abstract}

\section{Effect of Different Additives on the Rheological Behavior of Doughs of Plantain Flour of Dominico Hartón (Musa paradisiaca L.)}

\begin{abstract}
The objective of this work was to evaluate the effect of different additives in the rheological behavior of native plantain flour doughs for the formulation of a gluten-free functional flour. This behavior was analyzed through parameters such as elastic modulus ( $\left.G^{\prime}\right)$, viscous modulus ( $\left(G^{\prime \prime}\right)$, tangent of loss (tan $\delta$ ) and apparent viscosity. The choice of the most suitable formulation was done by means of a design of experiments completely randomized by comparison with the control (wheat flour). The egg protein, the emulsifier DATEM and xanthan gum generated viscoelastic parameters more similar to the control. The formulation of the functional flour dough that was most similar to the control was that with intermediate concentrations of the chosen additives. The conclusion is that the effect of the additives on the rheological behavior of the doughs of plantain flour depends on the chemical nature of the additive and the composition of the flour.
\end{abstract}

Keywords: additives; rheology; doughs; plantain flour; functional 


\section{INTRODUCCIÓN}

Según la base de datos de la Organización de las Naciones Unidas para la Alimentación y la Agricultura (FAOSTAT, 2018), para el año 2016, Colombia con 4.728 .179 de toneladas ocupó el cuarto lugar en producción mundial de plátano; de las cuales exporta el 3,6\%, cerca del 1,0\% se destina al consumo de la agroindustria y se estiman pérdidas equivalentes al $10 \%$ de la producción (Olmos, 2015), este porcentaje de pérdidas y de consumo, hace necesario promover investigaciones relacionadas con el procesamiento del fruto del plátano y su potencial de industrialización, haciendo necesario estudiar la relación entre las características funcionales y tecnológicas de las variedades de plátanos comerciales en Colombia y su aptitud para la elaboración de productos industriales. Las harinas son una excelente alternativa debido a que, además de ofrecer grandes beneficios de transformación, también aportan una gran variedad de posibilidades gastronómicas. Sin embargo, la ausencia de gluten en las harinas de plátano genera efectos tecnológicos negativos en su transformación. Para contrarrestar estos problemas, últimamente se ha investigado sobre la utilización de aditivos como hidrocoloides, proteínas sin gluten y otros ingredientes apropiados para harinas libres de gluten (Alvarez-Jubete et al., 2010; Caprilles y Áreas, 2014; Deora et al., 2014; Korus et al., 2015; O'Shea et al., 2014), (Sciriani et al, 2012), con el fin de mejorar la funcionalidad tecnológica de estas harinas. Una forma de evaluar el impacto de la adición de otras sustancias a las harinas es por medio de la reología de sus masas. Estas masas son alimentos fluidos que contienen compuestos de alto peso molecular que exhiben propiedades viscoelásticas; que son altamente sensibles a la temperatura, al contenido de agua y a la composición (origen del almidón, tipo de proteína, presencia de lípidos, etc.) (Ronda et. al, 2016) y por ello la adición de otras sustancias puede afectar estas propiedades positiva o negativamente. Además, la reología es una ciencia importante en el estudio de las propiedades de las masas, debido a que están relacionadas con su facilidad de manejo y su capacidad de desarrollo en los procesos de amasado, fermentación y horneado; siendo relevante en la determinación de la funcionalidad de los ingredientes para el desarrollo de productos (Torres et al., 2012).

Los emulsionantes se utilizan en la industria de la panadería debido a su capacidad de facilitar el proceso de emulsión de los ingredientes de la masa, reduciendo la tensión superficial entre dos fases inmiscibles (Gómez et al., 2003). Onyango et al., (2010) incorporó emulsionantes en formulaciones libres de gluten de pan hecho a base de almidón de yuca pregelatinizado y encontró que el emulsionante disminuyó la firmeza de la miga. La adición de proteínas en masas con ausencia de gluten, favorece el mejoramiento del sabor y la textura, y la disminución de la velocidad de endurecimiento de la miga (Kenny et al., 2001) además de aumentar su valor nutricional (Villanueva et al., 2014; Sarabhai et al., 2015). Estudios realizados por Gallagher et al., (2004) comprobaron que proteínas de suero de leche mejoran la capacidad de absorción de agua y las propiedades mecánicas de las masas libres de gluten. Los hidrocoloides suministran numerosas propiedades funcionales a los alimentos como dar cuerpo, gelificar, espesar y estabilizar (Sciarini, 2011). El uso de carboximetilcelulosa (CMC) en pan libre de gluten demostró que su inclusión a mayores concentraciones genera productos libres de gluten, con mayor firmeza, cohesividad, elasticidad y dureza (Martínez-Jiménez et al., 2014). La mezcla de goma xántan y guar en harinas de arroz con almidón de maíz y de papa, aumentó la retención de gas al interior de la matriz que ayudó a la estabilidad de la masa, durante el proceso de horneado (Mahmoud et al. 2013). Por otro lado, Jekle y Becker, (2014) demostraron que la microestructura del almidón influye notablemente en la apariencia, textura y estabilidad del producto final. Además, los sistemas de almidón pueden ser modificados por adición de diversos componentes como emulsionantes, almidones, hidrocoloides y proteínas, que interactúan con los polisacáridos de las harinas proporcionando estabilidad, propiedades densificantes, gelificantes y emulsionantes (BeMiller, 2011). No obstante, es importante aclarar que el efecto de los aditivos depende de su calidad y naturaleza, de la calidad de la materia prima y la disponibilidad de agua, siendo muy difícil predecir el efecto real de cada uno de ellos en las diferentes formulaciones (Sciarani et al., 2012). Teniendo en cuenta estas mejoras en las propiedades de las harinas al agregar aditivos, el objetivo de este trabajo fue evaluar el efecto de emulsionantes, almidones, hidrocoloides y proteínas, en el comportamiento reológico de masas de harina de plátano, con el fin de elegir los aditivos más adecuados (por comparación con la masa de harina de trigo) para la formulación de una harina de plátano con funcionalidad tecnológica.

\section{METODOLOGÍA}

Se trabajó con la variedad Dominico Hartón (Musa paradisiaca L.) suministrada por la Granja Integral Andalucía ubicada a $4^{\circ} 27^{\prime}$ latitud norte y $75^{\circ} 47^{\prime}$ longitud oeste, la temperatura y la humedad relativa promedio son $22^{\circ} \mathrm{C}$ y $85 \%$, la presión atmosférica es de $1018,63 \mathrm{hPa}$ y la altura es de $1200 \mathrm{msnm}$; los racimos fueron seleccionados y cosechados a las 16 semanas bajo los criterios técnicos manejados por la asociación de Musáceas del Quindío. Posteriormente se transportaron en canastillas y se almacenaron a una temperatura de $13^{\circ} \mathrm{C}$ y $90 \%$ de humedad. La obtención de la harina de plátano verde (HP) se llevó a cabo utilizando la metodología descrita por Lucas et al., (2013). La harina de trigo comercial (HT) fue adquirida en un mercado local de la ciudad de Armenia-Quindío-Colombia. 


\section{Selección de los aditivos}

Se incorporaron aditivos con la finalidad de mejorar la funcionalidad tecnológica de la HP. Para su selección se tuvo en cuenta los más utilizados en harinas libres de gluten (GF) para mejorar sus propiedades (Sciarini, 2011); como proteínas se utilizaron: proteína de leche en forma de suero parcialmente desmineralizado $40 \%$ (Compañía láctea agropecuaria lecheros de Young CLALDY S.A) y huevo entero salado pasteurizado deshidratado (OVOBRAND); los hidrocoloides de grado alimenticio fueron: goma xántan (GX), carraginato (CARR), alginato (ALG) y carboximetilcelulosa (CMC) suministrados por NORTHEAST PHARM. TRADING COMPANY; los emulsionantes fueron ésteres de monoglicéridos y del ácido diacetil tartárico (DATEM) y estearoil 2-lactilato de sodio (SSL) (CIMPA s.a.s.); finalmente se adicionó almidón de maíz (PROMIDEX) debido a que presenta las características más similares al almidón de trigo (Castellano-Jaramillo et. al, 2016). La elección de los diferentes aditivos para la preparación de la harina de plátano funcional, se realizó mediante la evaluación del comportamiento reológico de las masas adicionadas con cada uno de ellos, seleccionando el que presentó un comportamiento más parecido al control $(\mathrm{HT})$.

\section{Preparación de las masas}

Las cantidades de los aditivos seleccionados se adicionaron a la HP y se mezclaron basados en los protocolos descritos por Borges (2009). Para la preparación de $100 \mathrm{~g}$ de harina se agregó $60 \%$ de agua en base seca y las cantidades de los aditivos se definieron de acuerdo a la metodología propuesta por Sciarini et al, (2012) con algunas modificaciones, mediante la utilización de un diseño factorial con puntos centrales (Tabla 1), con la finalidad de definir las mejores concentraciones que generen un comportamiento reológico similar al control.

Tabla 1: Concentración de aditivos en masas de harina de plátano

\begin{tabular}{|c|c|c|c|c|c|c|c|c|c|}
\hline$N^{\circ}$ & $\begin{array}{c}\text { Proteína } \\
\text { de huevo } \\
(g)\end{array}$ & $\begin{array}{c}\text { Almidón } \\
\text { de maíz } \\
(g)\end{array}$ & $\begin{array}{c}\text { Goma } \\
\text { xántan } \\
(g)\end{array}$ & $\begin{array}{c}\text { DATEM } \\
(g)\end{array}$ & $N^{\circ}$ & $\begin{array}{c}\text { Proteína } \\
\text { de huevo } \\
(g)\end{array}$ & $\begin{array}{c}\text { Almidón } \\
\text { de maíz } \\
(g)\end{array}$ & $\begin{array}{c}\text { Goma } \\
\text { xántan } \\
(g)\end{array}$ & $\begin{array}{c}\text { DATEM } \\
(g)\end{array}$ \\
\hline F1 & 2,5 & 2,5 & 0,3 & 0,5 & F15 & 2,5 & 7,5 & 0,7 & 1,5 \\
\hline F2 & 7,5 & 2,5 & 0,3 & 0,5 & F16 & 7,5 & 7,5 & 0,7 & 1,5 \\
\hline F3 & 2,5 & 7,5 & 0,3 & 0,5 & F17 & 0,8 & 5,0 & 0,5 & 1,0 \\
\hline F4 & 7,5 & 7,5 & 0,3 & 0,5 & F18 & 9,2 & 5,0 & 0,5 & 1,0 \\
\hline F5 & 2,5 & 2,5 & 0,7 & 0,5 & F19 & 5,0 & 0,8 & 0,5 & 1,0 \\
\hline F6 & 7,5 & 2,5 & 0,7 & 0,5 & F20 & 5,0 & 9,2 & 0,5 & 1,0 \\
\hline F7 & 2,5 & 7,5 & 0,7 & 0,5 & F21 & 5,0 & 5 & 0,2 & 1,0 \\
\hline F8 & 7,5 & 7,5 & 0,7 & 0,5 & F22 & 5,0 & 5,0 & 0,8 & 1,0 \\
\hline F9 & 2,5 & 2,5 & 0,3 & 1,5 & F23 & 5,0 & 5,0 & 0,5 & 0,2 \\
\hline F10 & 7,5 & 2,5 & 0,3 & 1,5 & F24 & 5,0 & 5,0 & 0,5 & 1,8 \\
\hline F11 & 2,5 & 7,5 & 0,3 & 1,5 & F25 & 5,0 & 5,0 & 0,5 & 1,0 \\
\hline F12 & 7,5 & 7,5 & 0,3 & 1,5 & F26 & 5,0 & 5,0 & 0,5 & 1,0 \\
\hline F13 & 2,5 & 2,5 & 0,7 & 1,5 & F27 & 5,0 & 5,0 & 0,5 & 1,0 \\
\hline F14 & 7,5 & 2,5 & 0,7 & 1,5 & & & & & \\
\hline
\end{tabular}

\section{Análisis reológico}

Para la caracterización reológica de las masas se utilizó un reómetro dinámico (Marca Physica Anton Paar, MODELO MCR 301. Stuttgart, Alemania). Los ensayos que se realizaron fueron:

\section{Barrido de frecuencia}

Se realizó un barrido de frecuencia de $0,1 \mathrm{a} 100 \mathrm{~Hz}$ a una deformación desde 0,01 a 100\%, a una temperatura de $30^{\circ} \mathrm{C}$ con una geometría plato-plato $\left(60 \mathrm{~mm}\right.$ de diámetro y un ángulo de $\left.4^{\circ}\right)$. Después de la carga, la masa se dejó reposar durante 30 min para equilibrar tensiones y se aplicó una capa delgada de vaselina al borde de la muestra expuesta para evitar la pérdida de humedad durante las mediciones. En éstos ensayos, se obtuvieron los valores de módulo elástico $\left(G^{\prime}\right)$, viscoso $\left(G^{\prime \prime}\right)$ y tangente $\delta,\left(G^{\prime \prime} / G^{\prime}\right)$ para distintos valores de frecuencia $\omega$ (Korus et al., 2012). 


\section{Viscosidad aparente}

La viscosidad aparente se calculó como el cociente entre el esfuerzo de corte y la velocidad de corte o cizalla. El ensayo se desarrolló a una temperatura de $30^{\circ} \mathrm{C}$, y la velocidad de corte se delimitó entre 0,02 a $100 \mathrm{~s}^{-1}$, utilizando una geometría de plato rugoso de $40 \mathrm{~mm}$ de diámetro, a $1 \mathrm{~mm}$ de distancia del plato (Korus et al., 2012).

\section{Diseño experimental y análisis estadístico}

Una vez seleccionados los aditivos (almidón, proteínas, hidrocoloides y emulsionantes) que generaron el mejor efecto en el comportamiento reológico comparados con un control (masa de harina de trigo), se establecieron las concentraciones de cada uno de ellos mediante la utilización de un diseño central rotable, que consiste en un arreglo factorial fraccionado $2^{4}$ con 8 puntos axiales y tres repeticiones en el punto central, con la finalidad de definir las cantidades más adecuadas para mejorar tecnológicamente las masas. Los siguientes fueron los datos que se tuvieron en cuenta en el diseño:

$$
-\alpha,-1,0,1, \alpha \text { donde } \alpha=1.68
$$

Con el fin de analizar el efecto de la adición de los cuatro componentes en la formulación de las masas, se realizó un análisis de varianza, en donde se tomaron todos los tratamientos de manera conjunta dentro de un análisis de diseño completamente aleatorizado, seguidamente se realizó un análisis de componentes principales; con base en estos, se identificaron las variables más significativas o que influían en mayor medida en el factor. En la siguiente fase se realizaron pruebas de comparación de todos los tratamientos, en función de la masa preparada con harina de trigo y de acuerdo a las variables claves determinadas en el análisis de componentes principales. Estas pruebas se realizaron por medio de análisis comparativos de Tukey bajo una confiabilidad del 95\% utilizando el software estadístico Statgraphics versión Stratus.

\section{RESULTADOS Y DISCUSIÓN}

En la Tabla 2, se presentan los módulos viscoelásticos de la HP comparada con la HT. Las 2 harinas presentaron valores del módulo de almacenamiento (G') mayores que el módulo de pérdida (G") en toda la frecuencia de rango para todas las muestras, que indica un predominio de las propiedades elásticas por sobre las viscosas y que es común tanto en las harinas de trigo, como en las harinas libres de gluten (GF) que se comportan como sólidos viscoelásticos (Ronda et al., 2016; Korus et al., 2012). La HT presentó una disminución de la viscosidad aparente a medida que aumentó la velocidad de corte (Figura 1), este comportamiento es típico de fluidos pseudoplásticos y se debe a que el aumento del esfuerzo produce la destrucción de las interacciones entre las proteínas que forman la red de gluten con características viscoelásticas únicas (Burešová et al., 2014) lo cual se traduce en una mayor fluidez de la masa. Por el contrario la masa HP no presentó flujo viscoso, debido a que según lo reportado por Ronda et al., 2016 el índice de flujo depende de la formulación y específicamente del grado de hidratación de la masa; y en el caso de la harina nativa de plátano, al presentar un contenido bajo en proteínas es incapaz de formar una red viscoelástica al adicionar el agua, y por este motivo no fluye y se comportan como un sólido elástico.

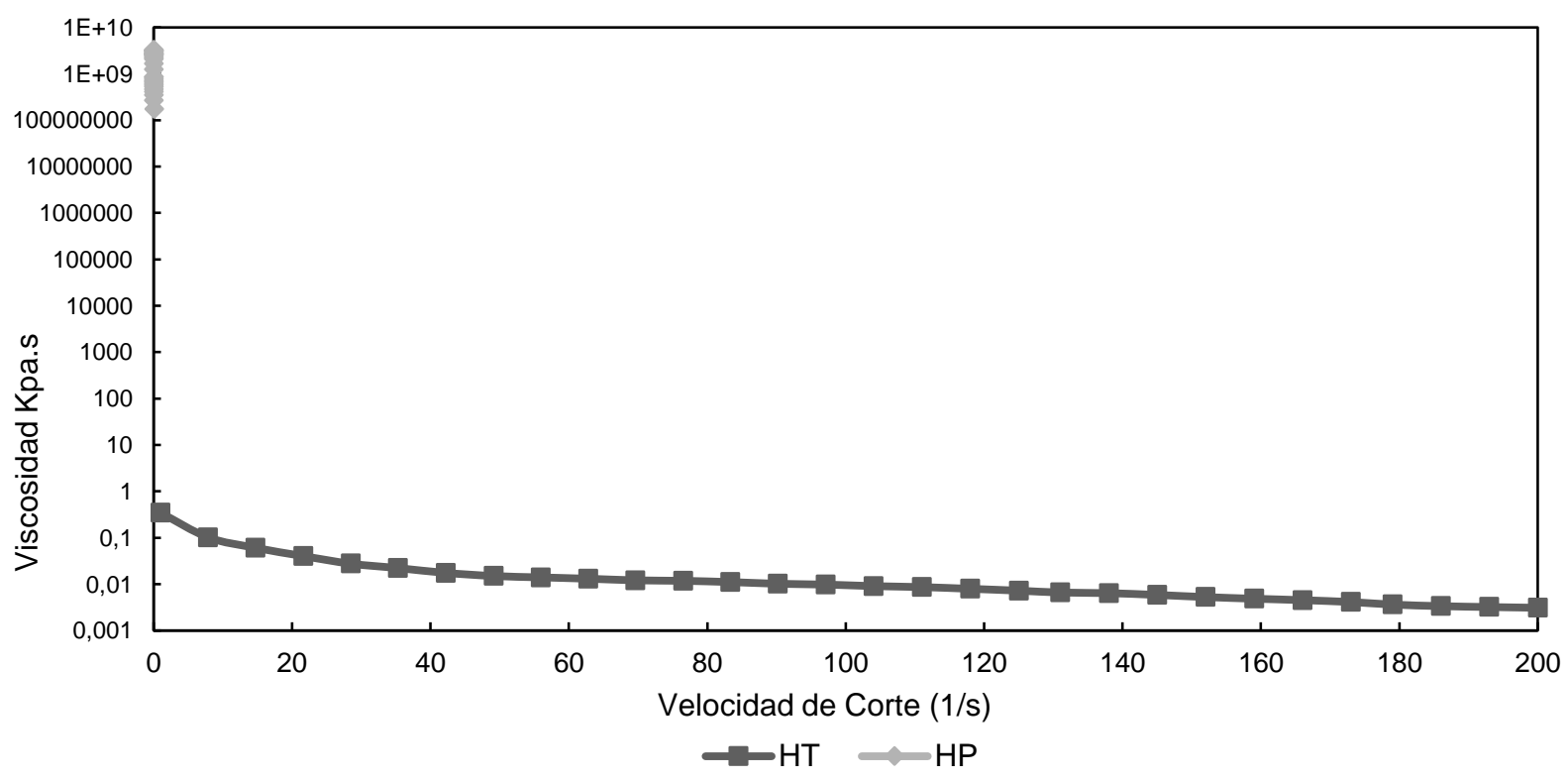

Fig.1: Variación de la viscosidad aparente en función de la velocidad de corte de masas preparadas con HT y HP 


\section{Efecto de la adición de proteínas sobre las propiedades de las masas}

En la Tabla 2 se observa que la adición de proteínas no cambió el comportamiento de los módulos, ya que el valor de G' se mantuvo mayor que G" en todo el espectro de las frecuencias analizadas. Por tanto, las masas presentan un comportamiento elástico que les confiere la capacidad de almacenar energía y es similar a lo reportado por Sciriani, (2011). Para los valores de módulo de almacenamiento y de pérdida se encontraron diferencias significativas $(P<0,05)$ entre las harinas plátano HPLS y HPHP, y en comparación con el control HT. Tal y como se observa el lactosuero provocó un aumento de estos dos parámetros mientras que el huevo en polvo favoreció su disminución, acercándolos a los valores de la harina de trigo. Para las masas preparadas con HT, se obtuvieron valores de G' y G" cercanos a $1 \mathrm{KPa}$ y para las masas de harina de plátano con proteínas, los valores fueron alrededor de $100 \mathrm{KPa}$; de acuerdo a estos resultados todas las masas se comportaron como un semisólido en todo el rango de frecuencias estudiadas, donde predomina el carácter elástico sobre el viscoso $\left(G^{\prime}>G\right)$.

Tal y como se observa en la Figura 2 con la adición de proteínas, las masas a base de harina de plátano presentaron un comportamiento similar al observado anteriormente para la HT. En primer lugar se observa que las viscosidades aparentes iniciales de la masas de harina plátano disminuyeron al orden de $1 \mathrm{KPa}$, aproximándose notablemente a la HT, además presentan flujo viscoso, lo que revela la formación de nuevas interacciones al agregar proteínas que permite que las masas fluyan; esto se debe a que según lo reportado por Sciarini, 2011 tanto las proteínas de leche como las de huevo tienen la capacidad de formar una red proteica similar a la del gluten y es por este motivo que se observa el adelgazamiento por cizalla típico de los fluidos pseudoplásticos, ya que a medida que avanza la velocidad de corte la estructura se relaja y se requiere de un menor esfuerzo para que las masas fluyan (Mezger, 2011). Estos cambios en la reología de las masas al agregar proteínas se deben a su capacidad de absorber e inmovilizar el agua (Parra, 2008).

La proteína que generó un comportamiento más parecido a la HT fue el huevo en polvo mientras que el lactosuero provoca una pérdida de estructura alrededor de los $110 \mathrm{~s}^{-1}$ que se evidencia por una caída repentina de la viscosidad de $50 \mathrm{~Pa}$ a $8 \mathrm{~Pa}$ aproximadamente, aunque esta disminución en la viscosidad de la HPLS se acerca más a los valores de la viscosidad aparente de la HT, el huevo en polvo genera una mayor estabilidad de la masa HPHP al no presentar caídas abruptas en la viscosidad, y además se asemeja al comportamiento de la HT en todo el rango de velocidades de corte. A pesar de que la composición y calidad de la proteína del lactosuero es bastante parecida a la del huevo (Parra, 2008), se encuentran diferencias en las cantidades de aminoácidos que pueden propiciar las interacciones que dan lugar a una red proteica más parecida a la del gluten. Es así como el huevo en polvo tiene una mayor cantidad de cisteína, metionina, valina, fenilalanina, histidina y triptófano que pueden favorecer interacciones hidrofílicas e hidrofóbicas (Parra, 2008) en la estructura de la red proteica y que repercuten en las propiedades funcionales de la H1HP haciéndola más parecida a la HT.

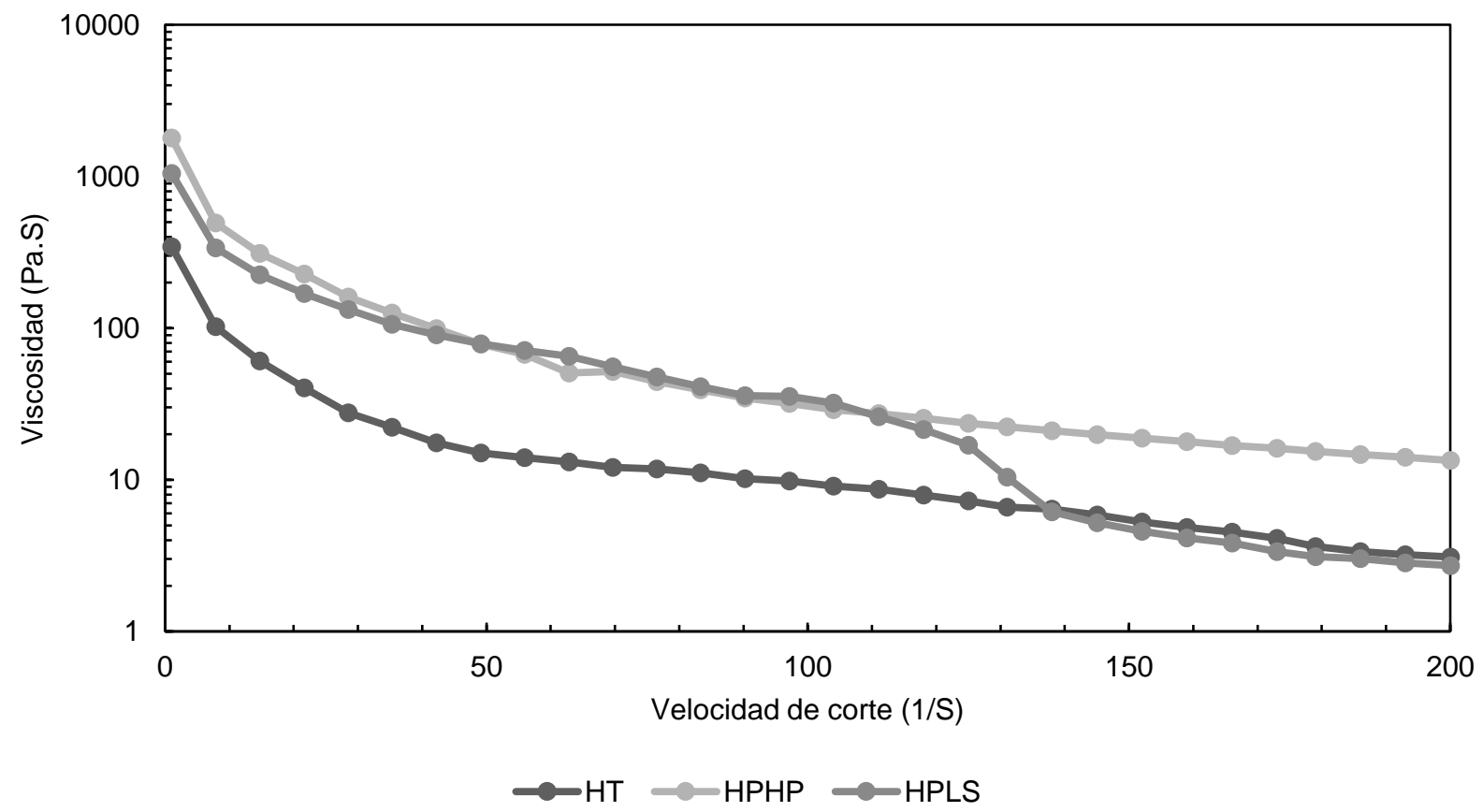

Fig.2: Variación de la viscosidad aparente en función de la velocidad de corte de masas preparadas con HT y HP con huevo en polvo (HPHP), lacto-suero (HPLS) 


\section{Efecto de la incorporación de emulsionantes}

En cuanto a la HP agregada con éster de monoglicéridos de ácido diacetil tartárico (DATEM), y estearoil 2lactilado de sodio (SSL), en la Tabla 2 se observa que la HP adicionada con emulsionante DATEM logró una mayor reducción con respecto a la harina con SSL de todos los parámetros viscoelásticos. Comparando las harinas adicionadas con la HP y la HT los valores de los módulos de almacenamiento y complejo aumentaron significativamente al agregar los emulsionantes, que como en el caso de la adición de proteínas a las masas, revelan una mayor capacidad de las estructuras para almacenar energía. La adición de DATEM a la HP generó un aumento significativo del módulo de almacenamiento, provocando un incremento de la tangente de pérdida que es indicativo de una mejora en la elasticidad de las masas, logrando de esta manera un comportamiento similar al control.

Los cambios en la reología de las masas de HP se deben a que la adición de emulsionantes; por su naturaleza anfipática, posibilita la interacción entre los lípidos, proteínas y el almidón, presentes en la harina nativa de plátano (Sciarini et al., 2012). Estos resultados son similares a lo reportado por Demirkesen et al., 2010 y Sciarini et al., 2012 para masas a base de harina de arroz adicionadas con DATEM y SSL. En el caso del DATEM la presencia de grupos hidroxilos en su estructura puede propiciar la interacción por puentes de hidrógeno con las proteínas y el almidón de la harina de plátano que estabilizaría mucho más la estructura que el SSL, haciendo la masa más elástica y confiriéndole propiedades funcionales más aproximadas a la harina de trigo.

Por otro lado, en la Figura 3 se muestra la viscosidad aparente de la HP adicionada con los emulsionantes y el control. Las harinas de plátano adicionadas presentaron un comportamiento de un fluido pseudoplástico que causó un adelgazamiento por corte, debido a la degradación de fricción de la amilosa y amilopectina que posee el almidón; a mayor velocidad de deformación, la destrucción de las interacciones provoca un reacomodo de la estructura de tal forma que las masas presentan una menor resistencia al flujo y por lo tanto presentan una menor viscosidad (Mezger, 2011). Es evidente que el DATEM confiere a la masa de plátano un comportamiento más aproximado al control que el SSL, a pesar de que al principio presenta una mayor viscosidad. Si se observa el perfil de flujo, la HPDATEM estructuralmente revela una mayor estabilidad que la HPSSL; que, aunque presenta una disminución de la viscosidad, muestra ciertas irregularidades en su comportamiento viscoso. Este hecho podría reforzar la teoría de que los grupos $\mathrm{OH}$ presentes en la estructura del DATEM generan una mayor estabilidad por la creación de puentes de hidrógeno con los componentes de la harina de plátano.

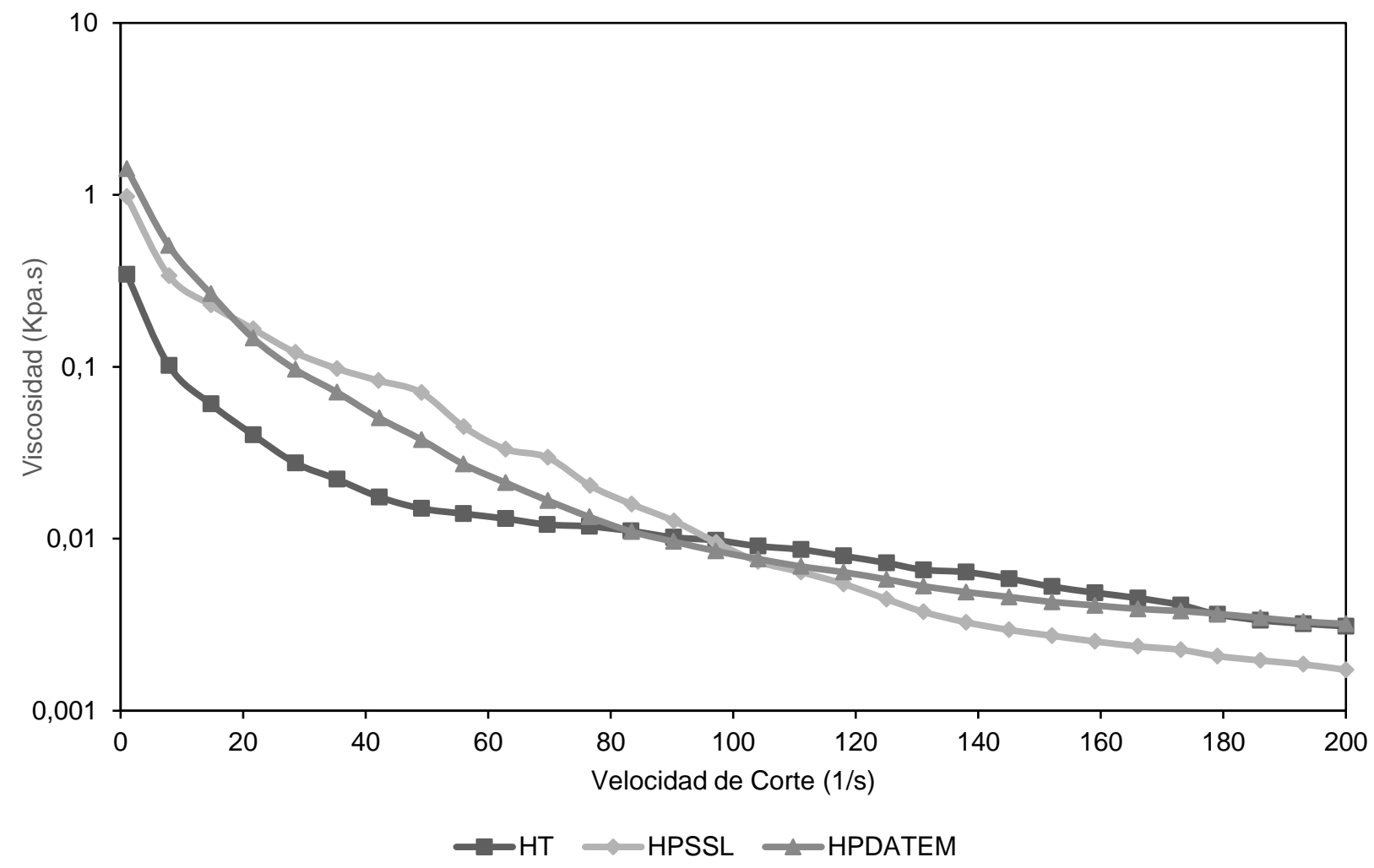

Fig. 3: Variación de la viscosidad aparente en función de la velocidad de corte de masas preparadas con HT y HP con éster de monoglicéridos de ácido diacetil tartárico (HPDATEM), y estearoil 2-lactilado de sodio (HPSSL) 


\section{Efecto de la incorporación de hidrocoloides sobre las propiedades de las masas}

En la Tabla 2 se evidencia que la adición de carragenato (CARR) y carboximetilcelulosa (CMC) a la HP generó un aumento más significativo de G' y G" para las masas. Este incremento del módulo de almacenamiento fue más acentuado para la HPCARR y por este motivo el factor de amortiguamiento disminuyó mientras que para la HPCMC aumentó. Por otro lado, la adición de alginato (ALG) y goma xántan (GX) a la harina de plátano provocó una disminución significativa del módulo de pérdida de las masas. El valor de G' para las dos masas fue alrededor de $69 \mathrm{Kpa}$, muy cercano a HP, mientras que para G" hubo una reducción más significativa en la HPGX que en HPALG. Este comportamiento provocó efectos diferentes en la tangente de pérdida, debido a que este parámetro disminuyó para la masa adicionada con GX mientras que aumentó para la adicionada con ALG.

De acuerdo a los resultados obtenidos, la adición de la GX generó un efecto significativo en los parámetros viscoelásticos de la masa de harina nativa de plátano que los acercó a la HT (control) tal y como se observa en la Figura 4. Según lo reportado por Sciarini et al., 2012 para masas a base de harina de arroz, la CMC y el ALG fueron los hidrocoloides que generaron una disminución de G' y G' mientras que la GX y el CARR tuvieron un efecto contrario; por otro lado, Lazaridou et al., 2007 obtuvieron un aumento de G' de las masas con la adición de CMC a la harina de arroz. Por tanto, se puede afirmar que dependiendo de la harina los hidrocoloides tienen un efecto diferente en las propiedades viscoelásticas de las masas. Para el caso del CARR y la GX la adición de hidrocoloides generó una disminución del factor de amortiguamiento (tan $\delta$ ) comparados con la HP, mientras que el ALG y la CMC generaron un aumento en el factor de amortiguamiento, siendo el ALG el valor que más se acercó al valor obtenido por la HT (control).

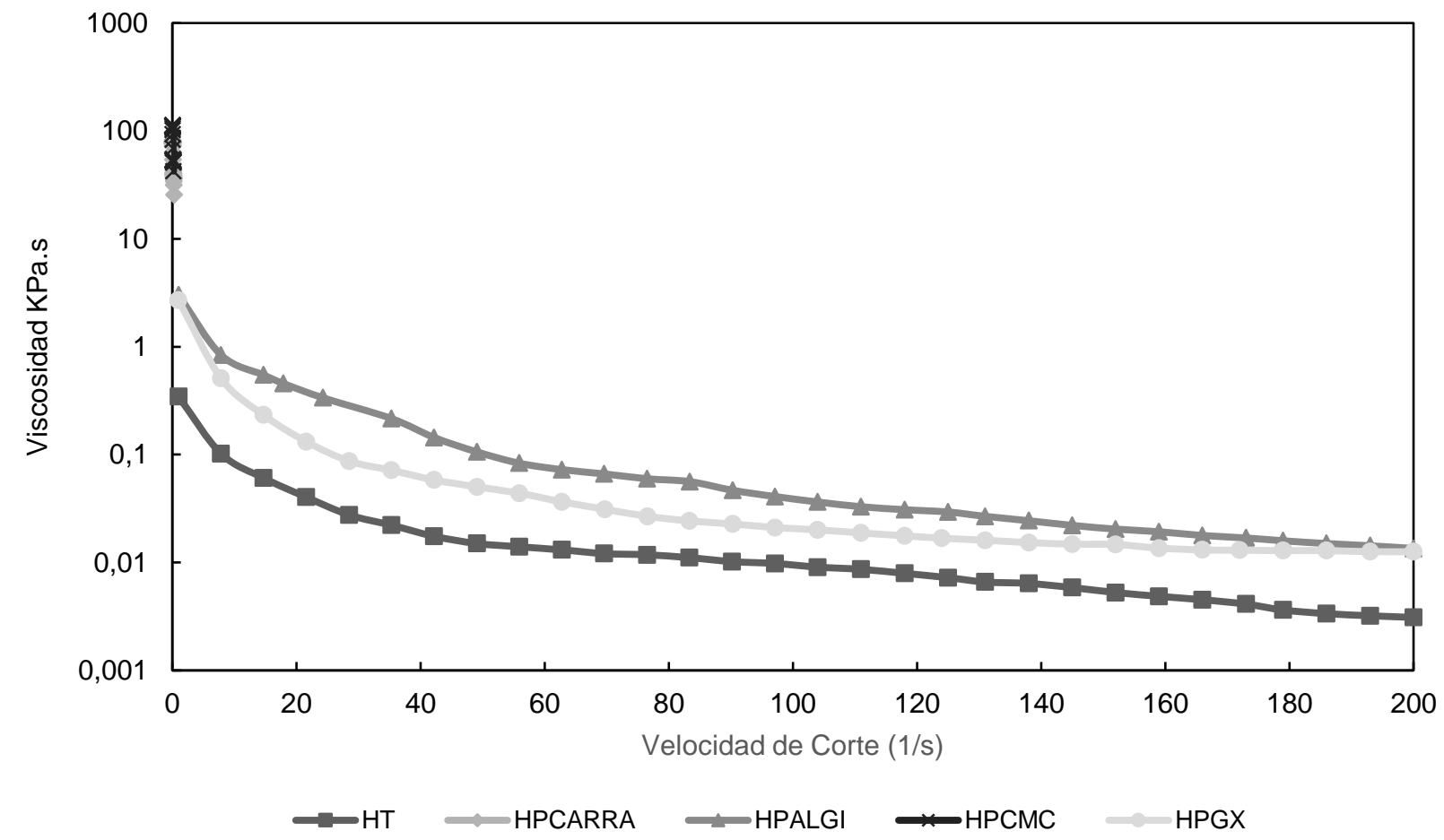

Fig. 4: Variación de la viscosidad aparente en función de la velocidad de corte de masas preparadas con HT y HP con carragenato (HPCARR), alginato (HPALG), carboximetilcelulosa (HPCMC) y goma xantan (HPGX)

En general los hidrocoloides pueden simular las propiedades viscoelásticas del gluten (Sanz et al., 2016), pero tal y como se observa en la Figura 4, la HPCARR y HPCMC no desarrollaron un perfil de flujo a medida que avanzó la velocidad de corte, comportándose como sólidos elásticos (Mezger, 2011). Este resultado es congruente con el aumento observado anteriormente en el módulo G' para las dos masas. Por otro HPALG y HPGX presentaron flujo viscoso bastante parecido al de la harina de trigo (control), como consecuencia de un menor valor en los módulos G' y G" de las masas cuando se les adicionó respectivamente los hidrocoloides, dando lugar a una estructura con propiedades viscoelásticas parecidas al gluten. En general la GX es el hidrocoloide que le confiere a la HP los valores más cercanos al control en los parámetros viscoelásticos (excepto en la tangente de pérdida), y consecuentemente obtuvo valores más parecidos de viscosidad aparente a la HT a medida que avanzó la velocidad de cizalla. Resultados diferentes fueron reportados por Sciarini, 2011 para harinas de arroz, debido a que todas las masas adicionadas con CARR, CMC, ALG y GX presentaron un comportamiento característico de fluidos pseudoplásticos, mostrando así que el efecto de los hidrocoloides depende de la composición de las harinas. 
Tabla 2: Módulos de almacenamiento (G'), de pérdida (G"), complejo (G*) y tangente de pérdida (tan $\delta)$ para masas preparadas con HT, HP y HP adicionadas con proteínas, emulsionantes e hidrocoloides

\begin{tabular}{|c|c|c|c|c|}
\hline Tratamiento & $G^{\prime}(\mathrm{Kpa})$ & $G^{\prime \prime}(\mathrm{KPa})$ & $\tan \delta$ & $G^{*}(\mathrm{KPa})$ \\
\hline HT & $2,16 \pm 0,08$ & $1,49 \pm 0,06$ & $0,69 \pm 0,00$ & $2,62 \pm 0,11$ \\
\hline HP & $64,00 \pm 1,20$ & $52,00 \pm 0,28$ & $0,81 \pm 0,01$ & $82,46 \pm 1,13$ \\
\hline HPLS & $238,00 \pm 0,00$ & $53,00 \pm 0,71$ & $0,22 \pm 0,22$ & $247,70 \pm 0,00$ \\
\hline HPHP & $240,00 \pm 0,00$ & $61,30 \pm 0,00$ & $0,26 \pm 0,25$ & $243,83 \pm 0,00$ \\
\hline HPDATEM & $118,00 \pm 0,00$ & $50,20 \pm 0,00$ & $0,42 \pm 0,00$ & $128,23 \pm 0,00$ \\
\hline HPSSL & $213,00 \pm 18,38$ & $46,45 \pm 5,30$ & $0,21 \pm 0,01$ & $218,01 \pm 18,38$ \\
\hline HPCARR & $403,00 \pm 19,80$ & $96,25 \pm 8,13$ & $0,23 \pm 0,01$ & $414,34 \pm 20,51$ \\
\hline HPALG & $68,95 \pm 1,48$ & $14,40 \pm 0,64$ & $0,20 \pm 0,00$ & $76,33 \pm 1,70$ \\
\hline HPCMC & $227,00 \pm 0,00$ & $98,00 \pm 0,00$ & $0,43 \pm 0,00$ & $247,25 \pm 0,00$ \\
\hline HPGX & $69,70 \pm 0,00$ & $32,75 \pm 0,00$ & $0,47 \pm 0,00$ & $71,17 \pm 0,00$ \\
\hline
\end{tabular}

Efecto de la incorporación de los aditivos seleccionados sobre las propiedades de las masas

Los resultados de los análisis reológicos de las 27 masas adicionadas se muestran en la Tabla 3. Se observó que la tendencia en el comportamiento del módulo elástico y viscoso de las masas se mantiene al agregar los aditivos a la vez en la HP en diferentes proporciones. Las masas se comportaron como un sólido viscoelástico con valores del módulo elástico (G') mayores que el módulo viscoso (G") en todo el rango de frecuencias y se mantuvieron relativamente constantes en las 27 muestras. Así mismo, todas las masas preparadas desarrrollaron flujo viscoso en todo el rango de velocidades de corte (gráfica no mostrada).

Tabla 3: Módulos viscoelásticos y tangente de pérdida para las masas preparadas con HP adicionadas

\begin{tabular}{|c|c|c|c|c|c|c|c|c|c|}
\hline$N^{\circ}$ & $\begin{array}{c}G^{\prime} \\
(\mathrm{Kpa})\end{array}$ & $\begin{array}{c}G^{\prime \prime} \\
(\mathrm{KPa})\end{array}$ & $\tan \delta$ & $\begin{array}{c}G^{*} \\
(\mathrm{KPa})\end{array}$ & $N^{\circ}$ & $\begin{array}{c}G^{\prime} \\
(\mathrm{Kpa})\end{array}$ & $\begin{array}{c}G^{\prime \prime} \\
(\mathrm{KPa})\end{array}$ & $\tan \delta$ & $\begin{array}{c}G^{*} \\
(\mathrm{KPa})\end{array}$ \\
\hline $\mathrm{F} 1$ & $254,5 \pm 1,23$ & $77,0 \pm 2,24$ & $0,3 \pm 0,00$ & $266,1 \pm 3,89$ & $\mathrm{~F} 15$ & $120,0 \pm 1,41$ & $33,9 \pm 2,76$ & $0,3 \pm 0,01$ & $124,7 \pm 1,41$ \\
\hline F2 & $225,0 \pm 1,46$ & $52,0 \pm 2,34$ & $0,2 \pm 0,00$ & $272,5 \pm 2,29$ & $F 16$ & $118,0 \pm 4,24$ & $30,0 \pm 2,47$ & $0,3 \pm 0,02$ & $121,7 \pm 5,66$ \\
\hline F3 & $267,5 \pm 2,92$ & $84,6 \pm 1,05$ & $0,3 \pm 0,00$ & $281,0 \pm 1,26$ & $F 17$ & $161,0 \pm 3,21$ & $44,1 \pm 1,08$ & $0,3 \pm 0,00$ & $166,9 \pm 4,74$ \\
\hline F4 & $159,0 \pm 1,42$ & $38,1 \pm 1,69$ & $0,2 \pm 0,01$ & $163,5 \pm 3,84$ & $F 18$ & $152,3 \pm 1,04$ & $36,2 \pm 2,74$ & $0,2 \pm 0,00$ & $156,6 \pm 6,03$ \\
\hline F5 & $123,0 \pm 0,07$ & $34,3 \pm 1,33$ & $0,3 \pm 0,01$ & $127,7 \pm 7,78$ & $F 19$ & $134,0 \pm 1,22$ & $30,7 \pm 1,40$ & $0,2 \pm 0,00$ & $137,5 \pm 3,79$ \\
\hline$F 6$ & $114,4 \pm 2,13$ & $34,5 \pm 1,69$ & $0,3 \pm 0,02$ & $119,4 \pm 2,33$ & $F 20$ & $136,7 \pm 1,02$ & $30,1 \pm 2,47$ & $0,2 \pm 0,00$ & $139,9 \pm 1,02$ \\
\hline$F 7$ & $136,0 \pm 3,11$ & $32,7 \pm 1,13$ & $0,2 \pm 0,02$ & $139,9 \pm 2,53$ & $F 21$ & $142,3 \pm 3,62$ & $35,7 \pm 1,76$ & $0,2 \pm 0,01$ & $146,8 \pm 2,62$ \\
\hline$F 8$ & $141,0 \pm 4,24$ & $34,4 \pm 0,85$ & $0,2 \pm 0,01$ & $145,1 \pm 3,54$ & $F 22$ & $118,3 \pm 1,69$ & $25,7 \pm 3,21$ & $0,2 \pm 0,01$ & $121,1 \pm 1,24$ \\
\hline$F 9$ & $147,0 \pm 5,66$ & $38,6 \pm 1,13$ & $0,3 \pm 0,00$ & $152,0 \pm 4,95$ & $F 23$ & $153,7 \pm 6,03$ & $31,7 \pm 2,45$ & $0,2 \pm 0,02$ & $156,9 \pm 6,03$ \\
\hline$F 10$ & $135,5 \pm 4,65$ & $35,0 \pm 1,17$ & $0,3 \pm 0,01$ & $140,0 \pm 3,36$ & $F 24$ & $119,7 \pm 1,37$ & $26,3 \pm 2,47$ & $0,2 \pm 0,01$ & $122,5 \pm 1,37$ \\
\hline$F 11$ & $167,5 \pm 3,54$ & $42,1 \pm 1,38$ & $0,3 \pm 0,02$ & $172,7 \pm 3,54$ & $F 25$ & $118,5 \pm 2,12$ & $26,5 \pm 0,21$ & $0,2 \pm 0,01$ & $121,4 \pm 2,12$ \\
\hline F12 & $139,0 \pm 5,66$ & $36,7 \pm 2,76$ & $0,3 \pm 0,01$ & $143,8 \pm 6,35$ & $F 26$ & $112,0 \pm 4,14$ & $23,1 \pm 1,48$ & $0,8 \pm 0,02$ & $114,4 \pm 4,14$ \\
\hline$F 13$ & $115,5 \pm 2,12$ & $30,2 \pm 0,64$ & $0,3 \pm 0,01$ & $119,4 \pm 1,41$ & $F 27$ & $116,5 \pm 4,95$ & $25,2 \pm 1,56$ & $0,2 \pm 0,01$ & $119,2 \pm 4,95$ \\
\hline$F 14$ & $137,0 \pm 2,28$ & $31,3 \pm 2,78$ & $0,2 \pm 0,01$ & $140,5 \pm 29,70$ & & & & & \\
\hline
\end{tabular}

Como ya se mencionó anteriormente este comportamiento de los módulos viscoelásticos es común para todos los tipos de masas tanto de trigo como libres de gluten; y se debe a que la adición de agua a la harina en la preparación de las masas, permite el desarrollo de una red viscoelástica (Ronda et al., 2016) que probablemente se formó por la interacción entre el almidón, las proteínas y los aditivos presentes en la harina de plátano adicionada. Aunque el almidón y el agua, pueden formar masas con propiedades mecánicas apropiadas, también interfieren en dichas interacciones la presencia de proteínas solubles, hidrocoloides y polisacáridos diferentes del almidón favoreciendo la formación de esta red (Ziobro et al., 2013). De esta manera se aumenta la calidad tecnológica de la harina y la masa de HP funcional alcanza un comportamiento similar al de la masa preparada con harina de trigo. En comparación con la masa de HP todas las masas 
presentaron un aumento significativo de G' que indica, como en el caso de la adición de los aditivos por separado a las masas, que se formaron nuevas interacciones en estas provocando un cambio en la estructura que permite almacenar una mayor cantidad de energía. Magaña et al. (2009), argumentan que la predominancia del G' puede sugerir una fuerza mayor en las masas, dadas por las proteínas, como ocurre en las masas preparadas con harina de trigo por la presencia de gluten.

F1, F2 y F3 presentaron un aumento significativo de G" con respecto a HP con la diferencia de que F1 y F3 aumentaron la tan $\delta$ mientras que la F2 bajó levemente este valor. El resto de las masas presentaron valores de G" significativamente menores que HP con algunas variaciones de la tan $\delta$. Al realizar un comparativo entre las formulaciones se observó que cuando se adiciona una mayor cantidad de proteína generalmente tiende a disminuir tanto el módulo elástico como el viscoso (Tabla 3), de igual forma ocurre cuando aumenta la cantidad del hidrocoloides. Se debe tener en cuenta que la disminución generalizada del comportamiento viscoso y el aumento del módulo elástico, son un indicativo de la creación de nuevas interacciones en las masas de harina de plátano adicionadas que forman una estructura continua con propiedades viscoelásticas. La F26 generó los valores más bajos para G', G" y G* de todas las formulaciones, que la acercan a la harina de trigo $(\mathrm{HT})$, y además presentó un flujo viscoso que presenta adelgazamiento por cizalla más estable que en las demás formulaciones. Esta formulación tiene concentraciones intermedias de proteína, almidón, hidrocoloide y emulsionante que se traducen en un buen equilibrio entre los dos módulos y por este motivo se considera la formulación más adecuada en términos reológicos para el desarrollo de una harina funcional a base de harina de plátano nativa.

\section{CONCLUSIONES}

De acuerdo con el análisis de los resultados obtenidos en esta investigación, se puede concluir que: 1) el efecto de los aditivos sobre el comportamiento reológico de las masas de harina de plátano depende tanto de la naturaleza química del aditivo, así como de composición de la harina 2) Todos los aditivos agregados a la harina de plátano, excepto el carragenato y la carboximetilcelulosa, favorecieron el flujo viscoso de las masas; revelando así la creación de diferentes interacciones entre los componentes de la harina. 3) Los aditivos que generaron un comportamiento reológico, más parecido a las masas de harinas de trigo, en la masa de harina de plátano fueron: El huevo en polvo, el DATEM y la goma Xantán. 4) La formulación 26 (F26) presentó los valores más cercanos de G', G", G* y flujo viscoso a la harina de trigo; y por tanto es la más adecuada para el desarrollo de la harina funcional de plátano libre de gluten.

\section{REFERENCIAS}

Alvarez-Jubete, L., A.K. Arendt y E. Gallaher, Nutritive Value of Pseudocereals and their Increasing Use as Functional Gluten-Free Ingredients, doi:10.1016/j.tifs.2009.10.014, Trends in Food Science \& Technology, 21, 106-113 (2010)

Bemiller, J.N., Pasting, Paste and Gel Properties of Starch-hydrocolloid Combinations, doi: 10.1016/j.carbpol.2011.05.064, Carbohidrate Polymers, 86, 386-423 (2011)

Borges, J.T. y D.S. Avaliação, Tecnológica de Farinha Mista de Trigo e de linhaça Integral e sua Utilização na Elaboração de pão de sal, Tesis Doctoral, Universidade Federal de Viçosa, Brasil (2009)

Burešová, I., S. Kráčmar, P. Dvořáková y T. Středa, The Relationship between Rheological Characteristics of Gluten-Free Dough and the Quality of Biologically Leavened Bread, doi:10.1016/j.jcs.2014.07.001, Journal of Cereal Science 60(2), 271-275 (2014)

Capriles, V.D. y J.A. Áreas, Novel Approaches in Gluten-Free Breadmaking: Interface between Food Science, Nutrition, and Health, doi:10.1111/1541-4337.12091, Food Science and Food Safety, 13, 871-890 (2014)

Castellano-Jaramillo, G., M.L. Rodríguez-Marín, A.D. Román-Gutiérrez y F. Guzmán-Ortiz, Evaluación de las Propiedades Fisicoquímicas de diferentes Cereales, doi: 10.29057/icbi.v3i6.573, Pädi Boletín científico de Ciencias Básicas e Ingeniería del ICBI, 3(6), (2016)

Demirkesen, I., B. Mert, G. Sumnu y S. Sahin, Rheological Properties of Gluten-Free Bread Formulations, doi: 10.1016/j.jfoodeng.2009.08.004, J. Food Eng, 96(2), 295-303 (2010)

Deora, N.S., A. Deswal y H.M. Mishra, Alternative Approaches Towards Gluten-Free Dough Development: Recent Trends, doi:10.1007/s12393-014-9079-6, Food Engineering Reviews 6(3), 89-104 (2014)

FAOSTAT, Base de Datos de la Organización de las Naciones Unidas para la Alimentación y la Agricultura, FAO (2018)

Gallagher, E., T.R. Gormley y E.K. Arendt, Recent Advances in the Formulation of Gluten-Free Cereal-based Product, doi: 10.1016/jitis.2003.09.012, Trends in Food Science and Technology, 15, 143-152 (2004)

Gómez, M., F. Ronda, C.A. Blanco y otros dos autores, Efect of Dietary Fibre on Dought Reology and Bread Quality, doi: 10.1007/s00217-002-0632-9, European Food Research and Technology, 216, 51-56 (2003)

Jekle, M., T. Becker, Wheat Dought Microstructure: the Relation between Visual Structure and Mechanical Behavior, doi: 10.1080/10408398.2012.65.64.76, Critical Reviews in Food Science and Nutrition, 53(3), 369-382 (2014) 
Kenny, S., K. Wehrle y M. Auty y E.K. Arendt, Influence of Sodium Caseinate and Whey Protein on Baking Properties and Rheology of Frozen Dough, doi: 10.1094/cchem.2001.78.4.458, Cereal Chemistry Journal, 78(4), 458-463 (2001)

Korus, J., L. Juszczak R. Ziobro y otros 3 autores, Defatted Strawberry and Blackcurrant Seeds as Functional Ingredients of Gluten-Free Bread, doi:10.1111/j.1745-4603.2011.00314.x, Journal of Texture Studies 43(1), 29-39 (2012)

Lazaridou, A., D. Duta, M. Papageorgiou, y otros 2 autores, Effects of Hydrocolloids on Dough Rheology and Bread Quality Parameters in Gluten-Free Formulations, doi: 10.1016/j.jfoodeng.2006.03.032, Journal of Food Engineering, 79(3), 10331047 (2007)

Lucas, J.C., B.D. Quintero y C.A. Cardenas, Caracterización de Harina y Almidón Obtenidos a partir de Plátano Guineo AAAea (Musa Sapientum L.), ISSN:0120-2812, Acta Agronómica, 62(2), 83-96 (2013)

Magaña, E.B., B. Ramírez, L.C. Platt y otros 2 autores, Caracterización Viscoelástica de Masas de Variedades de Trigos Suaves, ISSN: 0186-6036, Tecnol. Ciencia, Ed. (IMIQ) 24(1), 12-22 (2009)

Mahmoud, R.M., E.I. Yousif, M.G.E. Gadallah y otro autor, Formulation and Quality Characterization of Gluten-Free Egiptian Balady Flat Bread, doi: 10.1016/j.aoas.2013.01.004, Annals of Agricultural Science 58(1), 19-25 (2013)

Martinez-Jiménez, F., E. Rodríguez-Sandoval y M.S. Hernández, Influencia de Diferentes Emulsificantes en las Características Texturales de Pan Libre de Gluten, ISSN:, Rev. Fac. Nal. Agr. Medellín, 67(2), 634-636 (2014)

Mezger, T.G., The Rheology Handbook: For Users of Rotational and Oscillatory Rheometers, 2a Ed., 19-28, Vincentz Network, Hannover, Germany (2011)

O'shea, N., E. Arendt y E. Gallagher, State of the Art in Gluten-Free Research, doi: 10.1111/1750-3841.12479, Journal of food science, 79(6), 1067-1076 (2014)

Olmos, A.M., Cadena Productiva del Plátano Departamento de Casanare, Gobernación de Casanare: Secretaría de Agricultura, Ganadería y Medio Ambiente, (2015)

Onyango, C., C. Mutungi, G. Humbegen,y L. Meinolf, Rheological and Baking Characteristics of Batter and Bread Prepared from Pregelatinised Cassava Starch and Sorghum and Modified Using Microbial Transglutaminase, doi:10.1016/j.jfoodeng.2009.11.002, Journal of Engineering 97(4), 465-470 (2010)

Parra, R. A., Lactosuero: Importancias en la industria de Alimenticia, ISSN: 0304-2847, Rev.Fac.Nal.Agr.Medellín, 62(1), 4967-4982 (2008)

Ronda, F., S. Pérez-Quirce y M. Villanueva, Rheological Properties of Gluten-Free Bread Doughs: Relationship With Bread Quality, doi: 10.1016/B978-0-08-100431-9.0012-7, Advances in Food Rheology and Its Applications, 297-334 (2016).

Sanz, T., A. Salvador y M.J. Hernández, Creep-Recovery and Oscillatory Rheology of Flour-Based Systems, doi:10.1016/B978-0-08-100431-9.00011-5, Advances in Food Rheology and Its Applications, 277-295 (2016)

Sarabhai, S., D. Indrani, M. Vijaykrishnaraj y otros tres autores, Efect of Protein Concentrates, Emulsifiers and Textural and Sensory Characteristics of Gluten-Free Cookies and its Immunochemical Validation, doi:10.1007/s13197-014-14325, Journal of Food Science and Technology 52(6), 3763-3772 (2015)

Sciarini, L., Estudio del Efecto de Diferentes Aditivos sobre la Calidad y la Conservación de Panes Libres de Gluten, $1^{\text {a }}$ Ed., 34-36, La plata: Universidad Nacional de La Plata, La Plata, Argentina (2011)

Sciarini, L.S., P.D. Ribotta, A.E. Leon y G.T. Pérez, Incorporation of Several Additives into Gluten Free Breads: Effect on Dough Properties and Bread Quality, doi: 10.1016/j.jfoodeng.2012.03.011, Journal of Food Engineering 111, 590-597 (2012)

Torres, R., E.J. Montes, O.A. Pérez y R.D. Andrade, Influencia del Estado de Madurez sobre las Propiedades Viscoelásticas de Frutas Tropicales (Mango, Papaya y Plátano), doi: 10.4067/S0718-07642012000500012, Información Tecnológica 23(5), 115-124 (2012)

Villanueva, M., R.R. Mauro, C. Collar y R. Felicidad, Acidification of Protein-Enriched Rice Starch Doughs: Effects on Breadmaking, doi: 10.1007/s00217-014-2384-8, European Food Research and Technology 240(4), 783-794 (2014)

Ziobro, R., T. Witczak, L. Juszczak y J. Korus, Supplementation of Gluten-Free Bread with Non-Gluten Proteins. Effect on dought rheological properties and bread characteristic, doi:10.1016/j.foodhyd.2013.01.006, Food Hydrocolloids, 32(2), 213-220 (2013) 